\title{
Trombosis en el recién nacido
}

\author{
Thrombosis in newborn infants
}

\author{
Dra. Viviana Bacciedoni ${ }^{a, b}$, Dra. Myriam Attie ${ }^{c}$ y Dr. Hugo Donato ${ }^{d, e}$ \\ Comité Nacional de Hematología, Oncología y Medicina Transfusional.
}

\begin{abstract}
RESUMEN
La probabilidad de padecer trombosis es mucho mayor en el período neonatal que en cualquier otra etapa pediátrica. La labilidad del particular sistema hemostático del neonato, sumada a los múltiples factores de riesgo a que está expuesto y la presencia casi constante de catéteres, son responsables de este hecho. Las trombosis venosas son más frecuentes que las arteriales y ocurren principalmente en los miembros, la aurícula derecha y las venas renales. El accidente cerebrovascular puede ser causado por la oclusión del flujo arterial que llega al cerebro o del sistema de drenaje venoso de este. La púrpura fulminans es una patología de altísima gravedad, que debe ser considerada una emergencia médica y se debe a la deficiencia grave de proteína $\mathrm{C} \mathrm{o}$, menos frecuentemente, de proteína $\mathrm{S}$ o antitrombina. La mayoría de los episodios trombóticos tienen indicación de tratamiento anticoagulante, que se puede realizar con heparina no fraccionada y/o con heparina de bajo peso molecular. La púrpura fulminans requiere terapia de sustitución con proteína $\mathrm{C}$ y/o plasma fresco. El tratamiento trombolítico se realiza con activador tisular del plasminógenoy debe quedar reservado solo para aquellas trombosis cuya localización implique compromiso de vida o pérdida de un órgano o de un miembro.

Palabras clave: trombosis, recién nacido, heparina, anticoagulantes, terapia trombolítica.
\end{abstract}

http:/ / dx.doi.org/10.5546/aap.2016.159

\section{INTRODUCCIÓN}

En el primer mes de vida, la probabilidad de padecer complicaciones trombóticas es 40 veces superior a cualquier otra edad pediátrica, en especial en aquellos niños críticamente enfermos o que precisan catéter central. Este último es, sin duda, el factor de riesgo más importante para el desarrollo de tromboembolismo, tanto arterial como venoso. Aproximadamente, el $90 \%$ de los eventos tromboembólicos están asociados a algún tipo de catéter. ${ }^{1,2}$ Este favorece la ocurrencia de trombosis a través de distintos mecanismos, ya sea actuando aisladamente o en forma combinada entre ellos: provoca daño mecánico de la pared vascular y enlentecimiento o interrupción del flujo sanguíneo; está fabricado con material que es potencialmente trombogénico; y es utilizado para infundir sustancias que dañan la pared vascular. ${ }^{3}$ Los otros factores predisponentes son asfixia perinatal, prematuridad, trastornos cardíacos, sepsis, hipoxia y diabetes materna. Los estados protrombóticos hereditarios juegan un rol poco importante en este período. ${ }^{4}$

La incidencia global de tromboembolismo en recién nacidos hospitalizados es de aproximadamente 2,4 por 1000 admisiones. ${ }^{2,5}$ Se ha comunicado que el $1 \%$ de los neonatos con catéteres presentan síntomas sugestivos de trombosis, ${ }^{6}$ y se estima que la incidencia de trombosis asintomática asociada a catéteres está en el orden del $20 \%$ al 30\%. . $^{71}$

Su manejo adecuado se complica debido a que, generalmente, se extrapolan conductas de tratamiento del adulto. Sin embargo, en los últimos años, se han evidenciado importantes diferencias relacionadas con la edad en esta patología (epidemiología, pruebas diagnósticas, farmacocinética de los antitrombóticos), lo que está permitiendo comenzar a utilizar procedimientos diagnósticos y terapéuticos acordes a este período de la vida.

\section{CARACTERÍSTICAS DEL SISTEMA HEMOSTÁTICO DEL RECIÉN NACIDO}

El sistema hemostático del recién nacido presenta una serie de características especiales en relación con el adulto, que lo vuelven especialmente lábil. ${ }^{12}$ Síntesis disminuida de varias proteínas de coagulación (factores II, VII, IX, X, 
XI y XII, quininógeno de alto peso molecular, antitrombina III, proteínas S y C), actividad funcional alterada de otras (fibrinógeno, plasminógeno), depuración acelerada de factores y diferencias en el funcionalismo plaquetario son algunas de las particularidades que diferencian el sistema hemostático del neonato y del adulto. Además, tanto los estímulos recibidos durante el parto (acidosis, hipoxia, cambios térmicos, liberación de factores tisulares) como la exposición frecuente a traumas y manipulaciones determinan que el mecanismo de coagulación se encuentre en estado de mayor activación que el del adulto. Si bien los componentes del sistema de hemostasia comienzan a sintetizarse a la semana diez de gestación y sus niveles van luego aumentando gradualmente, los valores que alcanzan al momento del nacimiento difieren notablemente entre varios de ellos (Tabla 1).9,13-17

Con respecto a los factores de coagulación, los datos más relevantes son los siguientes::,16,18-24

- Los factores dependientes de la vitamina K (II, VII, IX, X) y los factores de contacto (XII, XI, precalicreína y quininógeno de alto peso molecular) están disminuidos en grado variable.

- El fibrinógeno y los factores V, VIII y XIII presentan valores similares al adulto.

- El nivel del factor Von Willebrand casi duplica el del adulto.

El tiempo necesario para que los factores disminuidos alcancen valores normales es variable y puede oscilar entre pocos días y varios meses. ${ }^{14-16,19,25,26}$ La mayoría alcanza el 80\% del valor del adulto a los seis meses.

Además, el fibrinógeno es cuantitativamente normal, pero presenta diferencias cualitativas, pues tiene mayor contenido de ácido siálico y una vida media menor. ${ }^{27-30}$

A pesar de estas alteraciones, en el neonato, se mantiene el equilibrio hemostático porque los inhibidores naturales también presentan diferencias respecto al adulto: ${ }^{13-15,19,25,26,31-35}$

- La antitrombina está reducida en un 50\% y las proteínas C y S, en un $60 \%$.

- La proteína S circula completamente libre (forma activa), pues el neonato no tiene $\mathrm{C} 4 \mathrm{~b}$, que liga la proteína $\mathrm{S}$.

TABLA 1. Valores promedio normales de factores procoagulantes, inhibidores y fibrinoliticos a las 24 horas de vida y tiempo aproximado necesario para alcanzar niveles normales

\begin{tabular}{lccc}
\hline & $\begin{array}{c}\text { Recién nacido } \\
\text { de término }\end{array}$ & $\begin{array}{c}\text { Recién nacido } \\
\text { pretérmino }\end{array}$ & $\begin{array}{c}\text { Tiempo para } \\
\text { alcanzar el valor normal }\end{array}$ \\
\hline $\begin{array}{c}\text { Procoagulantes: } \\
\text { Fibrinógeno\# }\end{array}$ & 2,83 & 2,43 & Al nacer \\
F. II* & 0,48 & 0,45 & $2-12$ meses \\
F. V & 0,72 & 0,88 & Al nacer \\
F. VII* & 0,66 & 0,67 & $2-12$ meses \\
F. VIII & 1,00 & 1,11 & Al nacer \\
F. IX*\# & 0,53 & 0,35 & $3-9$ meses \\
F. X* & 0,40 & 0,41 & $2-12$ meses \\
F. XI*\# & 0,38 & 0,30 & $1-2$ meses \\
F. XII*\# & 0,53 & 0,38 & $9-14$ días \\
F. XIII* & 0,79 & 0,70 & $4-5$ días \\
Precalicreína* & 0,37 & 0,33 & Más de 6 meses \\
HMWK & 0,54 & 0,49 & $2-3$ meses \\
F. Von Willebrand* & 1,53 & 1,36 & $5-6$ meses \\
Inhibidores: & & & 3 meses \\
Antitrombina*\# & 0,63 & 0,38 & Adulto \\
A2M*\# & 1,39 & 1,1 & $2-9$ meses \\
Proteína C*\# & 0,35 & 0,28 & 3 meses \\
Proteína S*\# & 0,36 & 0,26 & $6-12$ meses \\
Fibrinolíticos: & & & $3-4$ días \\
Plasminógeno* & 1,95 & 1,70 & $3-4$ días \\
Alfa 2 AP* & 0,85 & 0,78 & $3-4$ días \\
PAI*\# & 6,40 & 5,40 & 8,48 \\
TPA* & 9,60 & &
\end{tabular}

El fibrinógeno está expresado en $\mathrm{g} / \mathrm{L}$; todos los demás valores están expresados en $\mathrm{U} / \mathrm{mL}$.

F.: factor; HMWK: quininógeno de alto peso molecular; A2M: alfa-2-macroglobulina; Alfa 2 AP: alfa-2-antiplasmina;

PAI: inhibidor del activador tisular del plasminógeno; TPA: activador tisular del plasminógeno.

* Valores distintos al adulto; \# valores distintos en recién nacidos pretérmino y en nacidos a término. 
- La alfa-2-macroglobubilina está elevada a aproximadamente el doble del adulto.

La actividad fibrinolítica también está alterada, con niveles de plasminógeno reducidos al 50\%.

Muchas de estas diferencias recién descritas en todo el sistema hemostático son aún más marcadas en el prematuro.

\section{CUADROS CLÍNICOS}

\section{Trombosis venosas}

\section{Trombosis venosa profunda}

Es una patología frecuente en los pacientes internados en la Unidad de Cuidados Críticos, con incidencias informadas de $2 \%$ a $22 \%$. La trombosis de vena cava superior puede ser asintomática o manifestarse con edema de cuello, cara y/o zona superior del tórax, circulación colateral y eventual insuficiencia cardíaca aguda. ${ }^{36} \mathrm{La}$ trombosis en los miembros se puede manifestar con cambio de coloración, tumefacción, edema, dolor, aumento de temperatura y cianosis. En caso de compromiso de los miembros superiores, también puede presentarse como síndrome de vena cava superior. ${ }^{18,19,26,37,38}$

El síndrome postrombótico es una complicación a largo plazo que puede aparecer como consecuencia de trombosis neonatal, a partir del mes del episodio y hasta diez años después. Se caracteriza por edema crónico en las extremidades con decoloración de la piel, dificultad para la cicatrización de las heridas, úlceras en la piel y, frecuentemente, impotencia funcional. Aparece como resultado de la extravasación de hematíes y mediadores inflamatorios luego del daño de las válvulas venosas por el trombo. ${ }^{39}$

\section{Trombosis de vena renal}

Representa alrededor del 10\% de las trombosis venosas en el período neonatal. ${ }^{1,40}$ Es la trombosis más frecuente no relacionada con el catéter venoso central. Clínicamente, se puede presentar como masa palpable en flanco, hematuria, proteinuria, trombocitopenia, falla renal y/o hipertensión arterial. ${ }^{1,35} \mathrm{La}$ tríada clásica de masa palpable, hematuria y falla renal se ve solo en el $13 \%$ de los pacientes. ${ }^{41} \mathrm{Si}$ en su progresión alcanza la vena cava (alrededor del 50\% de los casos), puede, además, observarse edema, hipotermia y cianosis en los miembros inferiores. ${ }^{41-45}$ En aproximadamente un $25 \%$ de los casos es bilateral. ${ }^{5,46}$

Se presenta durante el primer mes de vida, por lo general, en los tres primeros días (67\% de los casos), pero también puede desarrollarse intraútero. ${ }^{1,13,25,32-35,42,43,47,48}$

Los factores de riesgo que se asocian con esta patología son asfixia, deshidratación, acidosis, hipotensión arterial, policitemia y diabetes materna.
El diagnóstico se realiza con ecografía doppler, que muestra pérdida de la diferenciación corticomedular renal o, preferentemente, con ecografía doppler color, en la que se observa la ausencia de flujo de la vena afectada. . $^{35,49-51}$

El tratamiento anticoagulante con heparina no fraccionada o de bajo peso molecular ha aumentado de modo considerable la supervivencia, que está actualmente en el orden del $85 \%,{ }^{1,40} \mathrm{y}$, además, evita la atrofia renal secuelar en $2 / 3$ de los pacientes. ${ }^{41}$

\section{Trombosis de aurícula derecha}

Representa aproximadamente el 6\% de las trombosis neonatales. El catéter venoso central está presente en casi todos los casos. ${ }^{52} \mathrm{La}$ presentación clínica es variable, con signos de insuficiencia cardíaca derecha, sepsis persistente, aparición súbita de soplo cardíaco, bradicardia, taquiarritmia o dificultad respiratoria. ${ }^{1,20}$ El método diagnóstico de elección es el ecocardiograma transtorácico.

Las complicaciones más graves son el tromboembolismo pulmonar, que se manifiesta como síndrome de dificultad respiratoria aguda, y el accidente cerebrovascular (ACV).

\section{Trombosis arteriales}

Las trombosis arteriales son, en general, una complicación iatrogénica de la cateterización de la arteria umbilical, las arterias periféricas o la arteria femoral. Su verdadera incidencia en recién nacidos se desconoce, ya que varía según el método utilizado para su evaluación: de $1 \%$ a $3 \%$ basado en signos clínicos, de $14 \%$ a $35 \%$ por ecografía y $64 \%$ por estudios angiográficos. ${ }^{1,53-56}$ La sintomatología depende de la localización del trombo y su extensión, que puede variar desde casi nula (mal funcionamiento del catéter) hasta isquemia masiva con potencial pérdida de miembro, hipertensión arterial con o sin insuficiencia renal (oclusión de la arteria renal), enterocolitis necrotizante (oclusión de la arteria mesentérica) o, incluso, embolia cerebral (por persistencia del foramen oval). ${ }^{1,57,58}$

La angiografía, que es el procedimiento de elección en niños mayores y adultos, no es utilizada en forma habitual en neonatos por los riesgos que implica el procedimiento. Es por eso por lo que, generalmente, se usa la ecografía doppler para confirmar el diagnóstico, aunque su verdadera especificidad no haya sido aún validada y, en algunos casos, pueda producir falsos resultados negativos. ${ }^{1,2,4,28}$

El tratamiento es difícil de decidir. La remoción del catéter es obligatoria, salvo aisladas excepciones. El riesgo/beneficio de 
utilizar anticoagulantes y trombolíticos no está establecido con claridad, por lo que debe ser evaluado en cada caso en particular. ${ }^{1}$ En líneas generales, si las trombosis no son oclusivas, pueden resolverse retirando el catéter, sin tratamiento anticoagulante; en cambio, si la oclusión es importante, el tratamiento inicial es heparina. ${ }^{4}$ En algunas situaciones específicas, con riesgo inminente de vida o de pérdida de un órgano o un miembro, se debe intentar el tratamiento trombolítico. ${ }^{1}$

\section{Accidente cerebrovascular}

El ACV puede ser debido a la oclusión del flujo arterial que llega al cerebro o del sistema de drenaje venoso de este -trombosis de senos venosos (TSV)-. La presentación característica es con convulsiones o letargia. La aparición de signos focales no es frecuente, y ocurre hemiparesia en menos del $25 \%$ de los niños con ACV isquémico y en menos del $10 \%$ de los casos de TSV.,40,59-64 También pueden observarse fontanela anterior tensa, diastasis de suturas de los huesos del cráneo y dilatación de las venas del cuero cabelludo en los casos de TSV. ${ }^{40,60,62,65}$

El diagnóstico confirmatorio de ACV isquémico se realiza mediante angiografía o angiorresonancia magnética. ${ }^{40,66}$ Para la TSV, es de elección la resonancia magnética, pero también brindan resultados satisfactorios la ecografía doppler transfontanelar y la ecografía convencional. ${ }^{67-70}$

La supervivencia sin secuela neurológica está en el orden de $33 \%$ a $50 \%$ aproximadamente. . $^{40,60,71,72}$

No se recomienda el tratamiento anticoagulante para el ACV, a menos que sea cardioembólico, en cuyo caso debe utilizarse heparina. ${ }^{40,73}$ Para la TSV sin áreas isquémicas extensas ni hemorragia intracerebral, se recomienda heparinización. Si presenta áreas isquémicas extensas o hemorragia intracerebral, se debe realizar un monitoreo cerebral y comenzar con anticoagulación si el trombo se extiende. ${ }^{40,73}$

\section{Púrpura fulminans}

Es una patología de altísima gravedad, que debe ser considerada una emergencia médica. Se manifiesta en niños con deficiencia grave de proteína $\mathrm{C}$ o, con mucha menos frecuencia, de proteína $S$ o antitrombina. ${ }^{74-76}$ Las lesiones se manifiestan en capilares de la piel, el cerebro y los riñones, debido a que la función de la proteína $\mathrm{C}$ se desarrolla fundamentalmente en la microcirculación.

La presentación clínica es característica. 40,74,75,77-81 Son niños que ya al nacer suelen presentar daño cerebral y/o oftálmico secundario a trombosis in- trauterina y que, en las primeras horas de vida, manifiestan el cuadro completo en forma catastrófica. Las lesiones cutáneas comienzan como pequeñas lesiones equimóticas, que gradualmente se van extendiendo en forma radial, cambian su coloración a rojo/negruzca, forman bullas y, por último, se vuelven necróticas y gangrenosas. Las lesiones se localizan, sobre todo, en las extremidades, pero pueden aparecer en cualquier otro sitio. La presencia de otras manifestaciones hemorrágicas, secundarias a coagulación intravascular diseminada, es casi constante. A veces, puede presentarse trombosis de grandes vasos.

El diagnóstico definitivo está basado en encontrar concentraciones bajas o no detectables de proteína $\mathrm{S}$ o $\mathrm{C}$, un alto grado de sospecha frente al cuadro clínico y el reconocimiento de un estado heterocigota en ambos padres. ${ }^{40,81,82}$

El cuadro cede con la administración de concentrado de proteína C (60 UI/ kg cada 6-8 horas, con posterior ajuste individual de dosis) $\mathrm{o}$, en su defecto, con plasma fresco congelado (10$20 \mathrm{ml} / \mathrm{kg}$ cada 8-12 horas). En la deficiencia de proteína $\mathrm{S}$, el tratamiento sustitutivo se efectúa con plasma fresco congelado..$^{79,83}$

El tratamiento se mantiene hasta que las lesiones se resuelvan completamente, lo cual ocurre dentro de las 6-8 semanas. ${ }^{40}$ El tratamiento a largo plazo se debe realizar con anticoagulación oral (manteniendo la razón internacional normatizada-RIN- entre 2,5 y 4,5) o con heparina de bajo peso molecular (HBPM), junto con terapia de reemplazo con proteína C (o S), de acuerdo con la sintomatología del paciente. ${ }^{40,80}$ La opción para la curación definitiva de la enfermedad es el trasplante hepático. ${ }^{40,75}$

\section{TRATAMIENTO}

\section{Manejo general de las trombosis}

Para la trombosis asintomática, se realizan cuidados de soporte y se recomienda monitorear el tamaño del coágulo. Si el trombo se asocia a catéter venoso central, este debe ser removido. Si el trombo progresa, se instaura el tratamiento anticoagulante. ${ }^{73}$

Para la trombosis sintomática, se recomienda el tratamiento con anticoagulantes y/o, raramente, trombolíticos. Los catéteres venosos centrales o umbilicales asociados a trombos deben ser removidos, si es posible después de tres a cinco días de anticoagulación. ${ }^{73,84}$ Los catéteres arteriales periféricos asociados a trombosis deben ser removidos rápidamente. ${ }^{73}$ La trombectomía quirúrgica rara vez está indicada en neonatos, ya que es un procedimiento limitado por el pequeño tamaño de los vasos y la inestabilidad clínica del paciente. ${ }^{85}$ 


\section{Tratamiento anticoagulante}

No se cuenta con suficientes estudios aleatorizados y controlados sobre anticoagulación en pediatría, por lo que los esquemas terapéuticos utilizados en estos pacientes se basan en estudios con pequeña cantidad de casos y guías adaptadas de tratamientos de adultos. Actualmente, las recomendaciones más utilizadas son las dadas por expertos y se basan en la evidencia, teniendo en cuenta que la mayoría tienen grado de evidencia 2 C. ${ }^{26,73,86}$

En los recién nacidos, existen dos inconvenientes adicionales. Por un lado, puede ser difícil obtener accesos venosos adecuados para realizar las extracciones necesarias para el control del tratamiento. Por el otro, debe considerarse que estos niños se alimentan con leches que contienen diferentes concentraciones de vitamina $\mathrm{K}$, lo que complica el uso de anticoagulantes orales.

Las drogas antitrombóticas más usadas son heparina no fraccionada y HBPM. Los trombolíticos, en general, no están indicados, excepto en situaciones de compromiso vital. Las nuevas drogas anticoagulantes se encuentran en evaluación y aún no pueden ser recomendadas para su uso en recién nacidos.

La duración óptima de la terapia no está claramente establecida, pero, en general, se recomienda administrarla de seis semanas a tres meses, según la situación clínica. ${ }^{73,87}$

Antes de comenzar el tratamiento antitrombótico, es conveniente chequear el tiempo de protrombina, el tiempo parcial de tromboplastina activada (TPTA), plaquetas y fibrinógeno. Durante el tratamiento anticoagulante, el recuento de plaquetas debe mantenerse por encima de $50 \times 10^{9} / \mathrm{L}$ y el fibrinógeno, mayor de $100 \mathrm{mg} / \mathrm{dL}$. Además, es conveniente realizar una ecografía cerebral previa, sobre todo en prematuros.

\section{Heparina no fraccionada}

La heparina no fraccionada actúa potenciando la acción de la antitrombina, que inactiva la trombina y el factor $X$ activado (FXa). Necesita, para ejercer su acción, una cantidad adecuada de antitrombina. Considerando que, al nacer, la concentración de antitrombina y la capacidad para generar trombina están fisiológicamente disminuidas (y más aún en el pretérmino), a veces, es necesaria su administración para alcanzar una anticoagulación adecuada. ${ }^{88,89}$

Se administra una dosis inicial de $75 \mathrm{UI} / \mathrm{kg}$ en bolo (10 minutos) y luego se mantiene con una dosis de $28 \mathrm{UI} / \mathrm{kg} / \mathrm{hora}$. El objetivo es lograr un nivel de anti-FXa de 0,3-0,7 UI/mL a las cuatro horas de la administración y una actividad de
TPTA de 1,5 a 2 veces el valor normal. ${ }^{88,90}$ Una vez alcanzado el rango terapéutico adecuado, se debe controlar cada $24 \mathrm{~h}$ con TPTA y recuento plaquetario.

Los efectos secundarios más importantes son el sangrado por exceso de dosis y la trombocitopenia inducida por heparina. En caso de sangrado, se debe suspender la droga y, si fuera necesario, administrar sulfato de protamina (1 mg de protamina cada 100 UI de heparina, en infusión endovenosa de 10 minutos).

Sus principales ventajas son el bajo costo y la rápida reversibilidad de su efecto si fuera necesario, ya que su vida media es de una hora.

\section{Heparina de bajo peso molecular}

Sus ventajas con respecto a la heparina no fraccionada son la administración subcutánea, la dosis cada 12-24 horas, la mínima necesidad de monitoreo, la respuesta más predecible y el menor riesgo de sangrado y de trombocitopenia inducida por la heparina. ${ }^{4}$

La HBPM más usada en neonatos es la enoxaparina. Para el tratamiento de trombosis, se recomienda la dosis de $1,5 \mathrm{mg} / \mathrm{kg}$ por vía subcutánea cada 12 horas, ${ }^{73}$ aunque actualmente existe evidencia de que, para alcanzar el rango terapéutico adecuado, la dosis útil está alrededor de $1,7 \mathrm{mg} / \mathrm{kg}$ en niños de término y $2 \mathrm{mg} / \mathrm{kg}$ en prematuros. ${ }^{91} \mathrm{El}$ objetivo es alcanzar un nivel de anti-FXa de 0,5-1 UI/mL. Para el uso como profilaxis, se recomienda la mitad de la dosis terapéutica con el fin de lograr un anti-FXa de $0,1-0,3 \mathrm{UI} / \mathrm{mL}$.

\section{Anticoagulantes orales}

Los anticoagulantes orales utilizados de manera habitual son antagonistas de la vitamina $\mathrm{K}$ y, por lo tanto, ejercen su acción disminuyendo la actividad funcional de los factores dependientes de la vitamina K (II, VII, IX y X). Considerando que el recién nacido tiene fisiológicamente niveles disminuidos de estos factores, el uso de estas drogas en el neonato se vuelve, entonces, problemático, sobre todo, por la gran variabilidad en la cantidad de vitamina K que tienen las distintas leches y que condiciona la respuesta al tratamiento: mientras que las fórmulas maternizadas contienen suplementos de vitamina K para prevenir la enfermedad hemorrágica por su deficiencia, lo que lleva a que los niños alimentados de esta manera sean relativamente resistentes a la medicación, la leche materna tiene baja concentración de vitamina $\mathrm{K}$ y, por lo tanto, estos niños son muy sensibles al tratamiento. ${ }^{1,73,92}$ Además, este tratamiento requiere monitoreos frecuentes, con la consiguiente dificultad para 
encontrar accesos venosos adecuados para la toma de muestras. ${ }^{1}$

De acuerdo, entonces, con la evidencia existente, el uso de antagonistas de la vitamina $\mathrm{K}$ en recién nacidos debe ser evitado en la medida de lo posible. ${ }^{1}$ Sin embargo, para tomar una decisión, también debe considerarse el hecho de que mantener una anticoagulación con HBPM durante varias semanas implica para el niño una conducta invasiva, que, en algunas oportunidades, lleva al no cumplimiento adecuado del tratamiento. Por lo tanto, tomando las precauciones del caso y realizando los controles con la frecuencia necesaria de acuerdo con las características de cada paciente, se puede indicar anticoagulación oral cuando va a ser de larga duración. Durante el tratamiento, se debe estar especialmente atento a las situaciones de variación en el tipo o la cantidad de la alimentación que recibe, a la ocurrencia de complicaciones infecciosas (en especial, gastrointestinales) y a la administración de antibióticos. ${ }^{3}$ En el caso de niños alimentados solo con leche materna, se puede compensar la baja ingesta de vitamina K provista por esta mediante la administración de dosis profilácticas de vitamina $K$ o de pequeñas cantidades diarias de fórmula maternizada. ${ }^{1,73}$

Se puede utilizar warfarina o acenocumarol. La dosis de comienzo recomendada es de 0,2 $\mathrm{mg} / \mathrm{kg} /$ día para alcanzar una RIN entre 2 y $3,^{40}$ aunque se ha visto que, en lactantes, la dosis útil promedio es de aproximadamente $0,33 \mathrm{mg} /$ $\mathrm{kg} /$ día. ${ }^{93}$ En caso de sangrado por exceso de dosis, se debe neutralizar su efecto mediante la administración de vitamina $\mathrm{K}^{40}$

Los anticoagulantes orales alternativos, como argatroban, bivalirudina o fondaparinux, todavía no tienen indicación en neonatos. ${ }^{94}$

\section{Tratamiento trombolitico}

Las drogas trombolíticas, en general, actúan promoviendo la conversión del plasminógeno a plasmina, la cual actúa sobre la fibrina, la degrada y produce la lisis del trombo. Dado que la actividad trombolítica puede estar reducida en estos pacientes por la concentración disminuida de plasminógeno, el efecto terapéutico de estas drogas puede estar limitado. La suplementación con plasminógeno por administración de plasma fresco congelado puede mejorar la actividad fibrinolítica.

Considerando el riesgo elevado de sangrado de este tratamiento, debe quedar reservado solo a aquellos niños que presenten trombosis cuya localización implique compromiso de vida o pérdida de un órgano o de un miembro. ${ }^{1,95,96}$ Está contraindicado en las siguientes situaciones: sangrado activo, cirugía mayor o hemorragia en los diez días previos, neurocirugía dentro de las tres semanas, evento asfíctico grave dentro de los siete días, procedimiento invasivo en los tres días anteriores, convulsiones en las últimas 48 horas, edad gestacional menor de 32 semanas. ${ }^{97}$

La droga de elección es el activador tisular del plasminógeno (Tissue Plasminogen Activator; TPA, por sus siglas en inglés). Se administra en infusión continua de $0,1-0,6 \mathrm{mg} / \mathrm{kg} /$ hora durante seis horas y puede usarse por vía central o periférica. ${ }^{73}$ No se dispone de pruebas específicas de laboratorio para definir el rango terapéutico ni para la monitorización. Debe realizarse un estricto control clínico, de laboratorio y radiológico. El aumento de tiempo de protrombina, TPTA, productos de la degradación de la fibrina (PDF) o dímero $\mathrm{D}$, así como la disminución del fibrinógeno, indican que hay respuesta al tratamiento trombolítico. Se recomienda mantener el fibrinógeno en valores por encima de $100 \mathrm{mg} / \mathrm{dL}$. Si hay sangrado, se recomienda administrar crioprecipitados (5-10 ml/kg) o fibrinógeno $y$, si hay riesgo vital, agregar antifibrinolíticos por vía endovenosa.

La mortalidad no está claramente definida y varía entre $1,2 \%$ y $13 \%$ de los casos tratados con TPA. ${ }^{98,99}$ La eficacia del tratamiento para obtener una resolución completa del coágulo es de $65 \%$ a $94 \% .^{100-102}$

\section{REFERENCIAS}

1. Greenway A, Massicotte MP, Monagle P. Neonatal thrombosis and its treatment. Blood Rev 2004;18(2):75-84.

2. SchmidtB, Andrew M. Neonatal thrombosis: report of a prospective Canadian and international registry. Pediatrics 1995;96(5 Pt 1):93943.

3. Donato H. Trastornos trombóticos. En: Donato H, Rapetti MC, eds. Hematología Neonatal. Buenos Aires: Fundasap; 2007.Págs.255-77.

4. Albisetti M, Andrew M, Monagle P. Hemostatic abnormalities. En: De Alarcón P, Werner E, eds. Neonatal hematology. New York: Cambridge University Press; 2005.Págs.310-48.

5. Hilario Barrio A, Gallego Herrero C, Miralles Molina M, Medina López C, et al. Trombosis venosa renal neonatal: diagnóstico precoz con ecografía Doppler y secuelas a largo plazo. Radiología 2009;51(6):583-90.

6. O'Neill JA Jr, Neblett WW 3rd, Born ML. Management of major thromboembolic complications of umbilical artery catheters. JPediatr Surg 1981;16(6):972-8.

7. Olinsky A, Aitken FG, Isdale JM. Thrombus formation after umbilical arterial catheterisation. An angiographic study. $S$ Afr Med J 1975;49(36):1467-70.

8. Oppenheimer DA, Carroll BA, Garth KE. Ultrasonic detection of complications following umbilical arterial catheterization in the neonate. Radiology 1982;145(3):667-72.

9. Horgan MJ, Bartoletti A, PolanskyS, Peters JC, et al. Effect of heparin infusates in umbilical arterial catheters on frequency of thrombotic complications. J Pediatr 1987;111(5):774-8.

10. Seibert JJ, Taylor BJ, Williamson SL, Williams BJ, et al. Sonographic detection of neonatalumbilical-artery thrombosis: clinical correlation. AJR Am J Roentgenol 1987;148(5):965-8.

11. Andrew M. Developmental hemostasis: relevance to newborns and infants. En: Nathan DG, Orkin SH, Oski FA, eds. Nathan and Oski's Hematology of Infancy and Childhood. $5^{\text {th }}$ ed. Philadelphia: WB Saunders; 1998.Págs.114-58. 
12. Donato H. Hemostasia normal en el feto y el recién nacido. En: Donato H, Rapetti MC, eds. Hematología Neonatal. Buenos Aires: Fundasap; 2007.Págs.195-205.

13. Nowak-Göttl U, Von Kries R, Göbel U. Neonatal symptomatic thromboembolism in Germany: two year survey. Arch Dis Child Fetal Neonatal Ed 1997;76(3):F163-7.

14. SchmidtB,Zipursky A. Thrombotic disease in newborninfants. Clin Perinatol 1984;11(2):461-88.

15. Khilnani P, Goldstein B, Todres ID. Double lumen umbilical venous catheters in critically ill neonates: a randomized prospective study. Crit Care Med 1991;19(11):1348-51.

16. Andrew M,DavidM,Adams M,AliK,etal.Venousthromboembolic complications (VTE) in children: first analyses of the Canadian Registry of VTE. Blood 1994;83(5):1251-7.

17. MonagleP,Adams M,Mahoney M,AliK, etal.Outcome of pediatric thromboembolic disease: a report from the Canadian Childhood Trombophilia Registry. Pediatr Res 2000;47(6):763-6.

18. Ament J, Newth CJ. Deep venous lines and thromboembolism. Pediatr Pulmonol 1995;20(6):347-8.

19. Marie I, Lévesque H, Cailleux N, Primard E, et al. Les thromboses veineuses profondes des membres supérieurs. À propos de 49 cas. Rev Med Interne 1998;19(6):399-408.

20. Bertrand M, Presant CA, Klein L, Scott E. Iatrogenic superior vena cava syndrome. A new entity. Cancer 1984;54(2):376-8.

21. Kramer SS, Taylor GA, Garfinkel DJ, Simmons MA. Lethal chylothoraces due to superior vena caval thrombosis in infants. AJR Am J Roentgenol 1981;137(3):559-63.

22. LeCoultreC,OberhänsliI,MossazA,BugmannP, etal.Postoperative chylothoraxin children: differences between vascular and traumatic origin. J Pediatr Surg 1991;26(5):519-23.

23. Andrew M, Paes B, Milner R, Johnston M, et al. Development of the human coagulation system in the full-term infant. Blood 1987;70(1):165-72.

24. Andrew M, Paes B, Milner R, Johnston M, et al. Development of the human coagulation system in the healthy premature infant. Blood 1988;72(5):1651-7.

25. Tinaztepe K, Buyan N, Tinaztepe B, Akkök N. The association of nephrotic syndromeand renal vein thrombosis: a clinicopathological analysis of eight pediatric patients. Turk J Pediatr 1989;31(1):1-18.

26. Mulvihill SJ, Fonkalsrud EW. Complications of superior versus inferior vena cava occlusion in infants receiving central total parenteral nutrition. J Pediatr Surg 1984;19(6):752-7.

27. Mitchell L, Chait P, Ginsberg J. Comparison of venography with ultrasound for the detection of venous thrombosis in the upper body in children: results of the PARKAA study. Blood 1999;94(Suppl 1):588a.

28. Edstrom CS, Christensen RD, Andrew M. Developmental aspects of blood hemostasis and disorders of coagulation and fibrinolysis in the neonatal period. En Christensen RD, ed. Hematologic problems of the Neonate. Philadelphia: Saunders; 2000.Págs.239-72.

29. KurekciE,KayeR,KoehlerM.Chylothoraxand chylopericardium:a complication of a central venous catheter. J Pediatr 1998;132(6):10646.

30. Barnes C, Newall F, Monagle P. Post-thrombotic syndrome. Arch Dis Child 2002;86(3):212-4.

31. Andrew M, Brooker LA. Hemostatic disorders in newborns. En Polin R, Fox W, Abman S, eds. Fetal and neonatal physiology. $2^{\text {nd }} \mathrm{ed}$. Philadelphia: WB Saunders; 1998.Págs.1803-33.

32. Reverdiau-MoalicP,DelahousseB,BodyG,BardosP, etal.Evolution of blood coagulation activators and inhibitors in the healthy fetus. Blood 1996;88(3):900-6

33. Nuss R, Hays T, Manco-Johnson M. Efficacy and safety of heparin anticoagulation for neonatal renal vein thrombosis. Am J Pediatr Hematol Oncol 1994;16(2):127-31.

34. Wright NB, Blanch G, Walkinshaw S, Pilling DW. Antenatal and neonatal renal vein thrombosis: new ultrasonic features with high frequency transducers. Pediatr Radiol 1996;26(9):686-9.

35. Andrew M, Monagle P, Brooker L. Thromboembolic complications during infancy and childhood. Hamilton BC: Decker Inc; 2000. Chapter 8, Thromboembolic complications in specific organ sites and pediatric diseases; Págs.231-76.

36. Ladino M, Ruiz-Esquide F, Guardia S. Trombosis venosa profunda en relación a un catéter central: edema neonatal en esclavina, un caso clínico. Rev Chil Pediatr 2001;72(4):340-4.

37. Rockoff MA, Gang DL, Vacanti JP. Fatal pulmonary embolism following removal of a central venous catheter. J Pediatr Surg 1984;19(3):307-9.

38. Derish MT, Smith DW, Frankel LR. Venous catheter thrombus formation and pulmonary embolism in children. Pediatr Pulmonol 1995;20(6):349-54

39. Sharathkumar AA,PipeSW.Post-thrombotic syndromein children: a single center experience. J Pediatric Hemat Oncol 2008;30(4):261-6.

40. Monagle P, Chan A, Massicotte P, Chalmers E, et al. Antithrombotic therapyinchildren: theSeventhACCPConferenceonAntithrombotic and Thrombolytic Therapy. Chest 2004;126(3 Suppl):645S-87S.

41. Zigman A, Yazbeck S, Emil S, Nguyen L. Renal vein thrombosis: a 10-year review. J Pediatr Surg 2000;35(11):1540-2.

42. Lalmand B, Avni EF, Nasr A, Ketelbant P, et al. Perinatal renal vein thrombosis: Sonographic demonstration. I Ultrasound Med 1990;9(8):437-42

43. Duncan BW, Adzick NS, Longaker MT, Edwards JR, et al. In utero arterial embolism from renal vein thrombosis with successful postnatal thrombolytic therapy. J Pediatr Surg 1991;26(6):741-3.

44. Brill PW, Jagannath A, Winchester P, Markisz JA, et al. Adrenal hemorrhageand renalvein thrombosisin thenewborn:MRimaging Radiology 1989;170(1 Pt 1):95-8.

45. Laplante S, Patriquin HB, Robitaille P, Filiatrault D, et al. Renal vein thrombosisinchildren: evidence of early flow recovery with Doppler US. Radiology 1993;189(1):37-42.

46. Marks SD, Massicotte MP, Steele BT, Matsell DG, et al. Neonatal renal venous thrombosis: clinical outcomes and prevalence of prothrombotic disorders. I Pediatr 2005;146(6):811-6.

47. Sanders LD, Jequier S. Ultrasound demonstration of prenatal renal vein thrombosis. Pediatr Radiol 1989;19(2):133-5.

48. Cozzolino DJ, Cendron M. Bilateral renal vein thrombosis in a newborn: a case of prenatal renal vein thrombosis. Urology 1997;50(1):128-31

49. Greene A,CromieWJ,GoldmanM.Computerizedbody tomography in neonatal renal vein thrombosis. Urology 1982;20(2):213-5.

50. Cremin BJ, Davey H, Oleszczuk-Raszke K. Neonatal renal venous thrombosis: sequential ultrasonic appearances. Clin Radiol 1991;44(1):52-5

51. Hibbert J, Howlett DC, Greenwood KL, MacDonald LM, et al. The ultrasound appearances of neonatal renalvein thrombosis. BrJRadiol 1997;70(839):1191-4.

52. AvilaML,SolanaCL,LauraJP,SuárezJ,etal.Trombectomíaauricular derecha en un recién nacido prematuro de muy bajo peso. Arch Arg Pediatr 2006;104(3):272-4

53. Downs JB, Chapman RL Jr, Hawkins IF Jr. Prolonged radialartery catheterization. An evaluation of heparinized catheters and continuous irrigation. Arch Surg 1974;108(5):671-3.

54. Hoar PF, Wilson RM, Mangano DT, Avery GJ $2^{\text {nd }}$, et al. Heparin bonding reduces thrombogenicity of pulmonary-artery catheters. N Engl J Med 1981;305(17):993-5.

55. American Association of Critical-Care Nurses. Evaluation of the effects of heparinized and nonheparinized flush solutions on the patency of arterial pressure monitoring lines: the AACN Thunder Project. Am J Crit Care 1993;2(1):3-15.

56. Cohen RS, Ramachandran P, Kim EH, Glasscock GF. Retrospective analysis of risks associated with an umbilical artery catheter system for continuous monitoring of arterial oxygen tension. J Perinatol 1995;15(3):195-8

57. Bryant BG. Drug, fluid, and blood products administered through the umbilical artery catheter: complication experiences from one NICU. Neonatal Netw 1990;9(1):27-32.

58. GlicksteinJS,RutkowskiM,SchachtR,FriedmanD. Renalblood flow 
velocity in neonates with and without umbilical artery catheters. J Clin Ultrasound 1994;22(9):543-50.

59. De Veber G. Canadian paediatric ischemic stroke registry: analysis of children with arterial ischemic stroke. Ann Neurol 2000;48(3):526a.

60. Barron TF, Gusnard DA, Zimmerman RA, Clancy RR. Cerebral venous thrombosis in neonates and children. Pediatr Neurol 1992;8(2):112-6.

61. Shevell MI, Silver K, O'Gorman AM, Watters GV, et al. Neonatal dural sinus thrombosis. Pediatr Neurol 1989;5(3):161-5.

62. Rivkin MJ, Anderson ML, Kaye EM. Neonatal idiopathic cerebral venous thrombosis: an unrecognized cause of transient seizures or lethargy. Ann Neurol 1992;32(1):51-6.

63. De Veber G, Adams M, Andrew M. Neonatal cerebral thromboembolism: clinical and radiographic features. Thromb Haemostas 1997;78(Suppl):725.

64. De Veber G, Andrew M, Adams C, Bjornson B, et al. Cerebral sinovenous thrombosis in children. NEngl JMed 2001;345(6):417-23.

65. DeVeberG,Andrew M.CanadianPediatricIschemicStrokeRegistry: analysis I. Pediatr Res 1994;35:379a.

66. LynchJK, Hirtz DG, De Veber G, Nelson KB. Report of the National Institute of NeurologicalDisordersandStrokeworkshoponperinatal and childhood stroke. Pediatrics 2002;109(1):116-23.

67. Macchi PJ, Grossman RI, Gomori JM, Goldberg HI, et al. High field MR imaging of cerebral venous thrombosis. J Comput Assist Tomogr 1986;10(1):10-5.

68. Zimmerman RA, Bogdan AR, Gusnard DA. Pediatric magnetic resonance angiography: assessment of stroke. Cardiovasc Intervent Radiol 1992;15(1):60-4.

69. MedlockMD, OliveroWC,HaniganWC, WrightRM, etal.Children withcerebral venousthrombosis diagnosed withmagneticresonance imaging and magnetic resonance angiography. Neurosurgery 1992;31(5):870-6.

70. Bezinque SL, Slovis TL, Touchette AS, Schave DM, et al. Characterization of superior sagittal sinus blood flow velocity using color flow Doppler in neonates and infants. Pediatr Radiol 1995;25(3):175-9.

71. DeVeberGA,MacGregorD,CurtisR,MayankS.Neurologicoutcome in survivors of childhood arterial ischemic stroke and sinovenous thrombosis. J Child Neurol 2000;15(5):316-24.

72. De Veber G, Adams M, Andrew M. Canadian Pediatric Ischemic Stroke Registry (analysis III). Can J Neurol Sci 1995;22(Suppl):S24a.

73. Monagle $\mathrm{P}$, Chan $\mathrm{AK}$, Goldenberg NA, Ichord RN, et al. Antithrombotic therapy in neonates and children: Antithrombotic Therapy and Prevention of Thrombosis, $9^{\text {th }}$ ed. American College of Chest Physicians Evidence-Based Clinical Practice Guidelines. Chest 2012;141(2 Suppl):e737S-e801S.

74. Adcock DM, Brozna J, Marlar RA. Proposed classification and pathologic mechanisms of purpura fulminans and skin necrosis. Semin Thromb Hemost 1990;16(4):333-40.

75. Marlar RA, Montgomery RR, Broekmans AW. Diagnosis and treatmentofhomozygous proteinCdeficiency.Reportof theWorking Party on Homozygous Protein CDeficiency of theSubcommittee on Protein $C$ and Protein S, International Committee on Thrombosis and Haemostasis. J Pediatr 1989;114(4 Pt 1):528-34.

76. Mahasandana C, Suvatte V, Chuansumrit A, Marlar RA, et al. Homozygous protein $S$ deficiency in an infant with purpura fulminans. J Pediatr 1990;117(5):750-3.

77. Auletta MJ, Headington JT. Purpura fulminans. A cutaneous manifestation of severe protein $\mathrm{C}$ deficiency. Arch Dermatol 1988;124(9):1387-91.

78. AdcockDM,HicksMJ.Dermatopathology of skinnecrosis associated with purpura fulminans. Semin Thromb Hemost 1990;16(4):283-92.

79. Marlar RA, Neumann A. Neonatal purpura fulminans due to homozygous protein $\mathrm{C}$ or protein $\mathrm{S}$ deficiencies. Semin Thromb Hemost 1990;16(4):299-309.

80. Andrew M, Monagle P, Brooker L. Thromboembolic complications during infancy and childhood. Hamilton BC: Decker Inc; 2000. Chapter3,Congenital prothrombotic disorders: presentationduring infancy and childhood; Págs.47-110.

81. Williams MD, Chalmers EA, Gibson BE. The investigation and management of neonatal haemostasis and thrombosis. Br J Haematol 2002;119(2):295-309.

82. Dreyfus M, Magny JF, Bridey F, Schwarz HP, et al. Treatment of homozygous protein $\mathrm{C}$ deficiency and neonatal purpura fulminans with a purified protein C concentrate. NEngl JMed 1991;325(22):15658.

83. Chalmers EA. Neonatal thrombosis. J Clin Pathol 2000;53(6):419-23.

84. Edstrom CS,ChristensenRD. Evaluationand treatmentof thrombosis in the neonatal intensive care unit. Clin Perinatol 2000;27(3):623-41.

85. LinPH,Dodson TF, BushRL, Weiss VJ,etal.Surgicalintervention for complications caused by femoral artery catheterization in pediatric patients. J Vasc Surg 2001;34(6):1071-8.

86. Altuna D. Trombosis en pediatría. Hematología (B Aires) 2013;17:38-43.

87. Dix D, Andrew M, Marzinotto V, Charpentier K, et al. The use of low molecular weight heparin in pediatric patients: a prospective cohort study. J Pediatric 2000;136(4):439-45.

88. Andrew M, Marzinotto V, MassicotteP, Blanchette V, et al. Heparin therapy in pediatric patients: a prospective cohort study. Pediatric Res 1994;35(1):78-83.

89. Andrew M, Ofosu F, Schmidt B, Brooker L, et al. Heparin clearance and ex vivo recovery in newborn piglets and adult pigs. Thromb Res 1988;52(6):517-27.

90. Schmidt B, Buchanan MR, Ofosu F, Brooker L, et al. Antithrombotic properties of heparinin aneonatal pigletmodel of thrombin-induced thrombosis. Thromb Haemost 1988;60(2):289-92.

91. Streif W, Goebel G, Chan AK, Massicotte MP. Use of low molecular mass heparin (enoxaparin) in newborn infants: a prospective cohort study of 62 patients. Arch Dis Child Fetal Neonatal Ed 2003;88(5):F36570.

92. Andrew M, Monagle P, Brooker L. Thromboembolic complications during infancy and childhood. Hamilton BC: Decker Inc; 2000. Chapter 9, Oral anticoagulation therapy in pediatric patients; Págs.278-356.

93. Streif W, Andrew M, Marzinotto V, Massicotte P, et al. Analysis of warfarin therapy in pediatric patients: a prospective cohort study of 319 patients. Blood 1999;94(9):3007-14.

94. Young G. Old and new antithrombotic drugs in neonates and neonates and infants. Semin Fetal Neonatal Med 2011;16(6):349-54.

95. Monagle P, Michelson AD, Bovill E, Andrew M. Antithrombotic therapy in children. Chest 2001;119(1 Suppl):344S-370S.

96. Ries M, Easton RL, Longstaff C, Zenker M, et al. Differences between neonates and adults in tissue-type-plasminogen activator (t-PA) catalyzed plasminogen activation with various effectors in carbohydrate sequences of fibrinogen chains. Thromb Res 2001;103(3):173-84.

97. Manco-Johnson MJ, Grabowski EF, Hellgreen M, Khemali AS, et al. Recommendations for tPA thrombolysisinchildren. Onbehalf of the ScientificSubcommittee onPerinataland Pediatric Thrombosis of the ScientificandStandardization Committee of the InternationalSociety of Thrombosis and Haemostasis. Thromb Haemost 2002;88(1):157-8.

98. ZenzW,ArltF,SodiaS, Beghold A.Intracerebralhemorrhageduring fibrinolytic therapy in children: a review of the literature of the last thirty years. Semin Thromb Hemost 1997;23(3):321-32.

99. Farnoux C, Camard O, Pinquier D, Hurtaud-Roux MF, et al. Recombinant tissue-type plasminogen activator therapy of thrombosis in 16 neonates. J Pediatr 1998;133(1):137-40.

100. Zenz W, Muntean W, Beitzke A, Zobel G, et al. Tissue plasminogen activator (alteplase) treatment for femoral artery thrombosis after cardiac catheterisation in infants and children. Br Heart $J$ 1993;70(4):382-5.

101. Andrew M, Monagle P, Brooker L. Thromboembolic complications during infancy and childhood. Hamilton BC: Decker Inc; 2000. Chapter 10, Thrombolytic therapy; p. 357-84.

102. Browne M, Newall F, Campbell J, Savoia HF, et al. Thrombolytic therapy with tissue plasminogen activator (tPA), analysis of safety and outcome in children. J Thromb Haemost 2003;1(Suppl 1):P1488. 